\title{
Evaluating Implementation Contexts, Mechanisms, and Outcomes: A Normalization Process Theory Coding Manual for Qualitative Research and Instrument Development
}

Carl May ( $\square$ carl.may@lshtm.ac.uk)

London School of Hygiene and Tropical Medicine Faculty of Public Health and Policy

https://orcid.org/0000-0002-0451-2690

Bianca Albers

University of Zurich: Universitat Zurich

Mike Bracher

University of Southampton

Tracy L Finch

University of Northumbria at Newcastle: Northumbria University

Anthony Gilbert

Royal National Orthopaedic Hospital NHS Trust

Melissa Girling

Northumbria University

Kathryn Greenwood

University of Sussex

Anne MacFarlane

University of Limerick

Frances Mair

University of Glasgow

Christine M May

LSHTM: London School of Hygiene \& Tropical Medicine

Elizabeth Murray

UCL: University College London

Sebastian Potthoff

Northumbria University

Tim Rapley

Northumbria University 
Keywords: parsimonious, coding manuals, generalizable coding manuals, Normalization Process Theory, Context-Mechanism-Outcomes

Posted Date: September 15th, 2021

DOI: https://doi.org/10.21203/rs.3.rs-870733/v1

License: (1) This work is licensed under a Creative Commons Attribution 4.0 International License. Read Full License 


\section{Abstract}

Background. Qualitative studies, especially those conducted by teams of researchers, may benefit from clearly structured, parsimonious, coding manuals. The process of creating rigorous and robust coding manuals for individual studies is rarely described, and generalizable coding manuals are rare. Normalization Process Theory (NPT) provides conceptual tools to facilitate understanding of the dynamics of adoption, implementation, and sustainment of socio-technical and organizational innovations. As a widely used theory, a generalizable coding manual would be of utility to implementation researchers

Objectives. To make the application of NPT simple for the user, to describe the development of a coding manual for qualitative content analytic studies using NPT, and present this for wider use.

Method. Concept Selection and Structuring. Qualitative Content Analysis of selected published papers and interview transcripts.

Results. All identifiable theoretical concepts $(n=149)$ embedded in papers and chapters that developed NPT between 2006 and 2020 were identified and extracted from their texts. Overlapping, ambiguous, and duplicate versions of concepts were eliminated, as were concepts derived from other theories. This left 38 core concept definitions. These were piloted in coding of qualitative transcripts collected in two implementation studies, and by collaboratively coding papers collected for a systematic review of implementation studies. At the end of this process, a further process of elimination of overlapping or ambiguous concepts was undertaken leaving 12 primary NPT concepts.

Conclusion. The process of coding manual resulted in the presentation of NPT concepts according to the Context-Mechanism-Outcomes configuration of realist evaluation research. A coding manual for NPT that is in accordance with realistic evaluation research was successfully produced and is now freely available to researchers who wish to use NPT in primary and secondary research that employs qualitative methods.

\section{Contribution To The Literature}

- Normalization Process Theory is widely used to design complex interventions and understand the dynamics of implementation processes and their outcomes.

- Coding manuals simplify theories and reduce the cognitive burden on teams performing qualitative analysis together.

- This paper describes the methods of developing a qualitative coding manual for Normalization Process Theory, and it presents the manual ready to use.

- The coding manual simplifies Normalization Process Theory for the user, and links it to the conceptual tools of Realist Evaluation. 


\section{Background}

Any researcher who wishes to become proficient at doing qualitative analysis must learn to code well and easily. The excellence of the research rests in large part on the excellence of the coding.

\section{Anselm Strauss [1]}

Qualitative Content Analysis (QCA) has become an important research method in recent years [2]. Although QCA is often seen primarily as a deductive approach to qualitative data analysis, in practice almost all qualitative methods involve some degree of content analysis. This is because qualitative research focuses on the identification, characterization and interpretation of empirical regularities or deviant cases in natural language data. Such an approach is reflected in the standard definitions of QCA. For example, Hsieh and Shannon [3], define QCA as, 'a research method for the subjective interpretation of the content of text-data through the systematic classification process of coding and identifying themes or patterns.' While for Patton [4], QCA is defined as, 'any qualitative data reduction and sensemaking effort that takes a volume of qualitative material and attempts to identify core consistencies and meanings.' As QCA has become more widely used, so too have coding frameworks and manuals that define the ways that data are identified, categorized, and characterized within a study. Qualitative researchers are, for example, encouraged to develop guidance that explicates each code, its definition and the 'rules' for what kind of data are, or are not relevant to each code [5].

Coding involves a process of data transformation. Most forms of qualitative analysis involve identifying and characterizing a set of concepts that are found to be salient to a research problem. The process of data transformation that follows from using a coding manual has two advantages. It enables researchers to manage the cognitive burden of searching for and handling multiple concepts, and thus enables them to manage a greater cognitive burden of interpretation. Researchers can therefore transparently share nuanced meanings and novel connections that are represented in any given corpus of data. Within research teams, coding manuals support the quality and rigor of coding by providing 'rules' that are employed by each team member and, in this way, can ensure the consistency of coding. Parsimony can be important too: more is not necessarily better in qualitative investigation and analysis. Reducing the number of codes to those that represent core concepts can be understood as what Adams et al [6], in a different context, have called subtractive transformation.

\section{The purpose of this paper}

Despite their value, the process of creating rigorous and robust coding manuals for individual studies is rarely described, and generalizable coding manuals are rare. These would be particularly useful for applying theoretical constructs that are used by qualitative researchers. In this paper, we fill this gap. We describe the purposes, methods of development, and potential application of a coding manual that translates Normalization Process Theory (NPT) into an easily usable framework for qualitative analysis. NPT provides a set of conceptual tools that support understanding and evaluation of the adoption, 
implementation, and sustainment of socio-technical and organizational innovations [7-11]. It is supported by empirical studies using both qualitative and quantitative methods, and by systematic reviews that have explored its value in different research domains [12-14]. As a widely used theory, a generalizable coding manual would be of value to implementation researchers from a range of disciplines. This paper also serves to make the coding manual freely available to other researchers.

The coding manual performs four important functions. First, it identifies and defines the core concepts of the theory, shows how they relate to each other, and demonstrates how they can be used to transparently categorize qualitative data. Second, it provides a clear and consistent set of definitions of core concepts in NPT that can be used when QCA is being conducted by teams working together on large bodies of data and, sharing-and sometimes checking-each other's analytic work. Third, it offers a starting point for multimodal analysis, (for example, in evaluations employing different qualitative research techniques), and a framework for directed content analysis (for example, in qualitative evidence syntheses). Finally, as software for computational hermeneutics [15] becomes more widely available and practically workable, a coding framework such as this can also be integrated into the development of topic modelling instruments and algorithms.

\section{Methods}

NPT is one of the most widely used implementation theories, and is often used to inform qualitative research on the implementation of socio- technical and organizational innovations in healthcare and other domains. As it has developed, has moved through multiple iterations, and its conceptual foundations have become more complex. These iterations have resulted from:

a. Theory-building: the construction and interpretation of core concepts of NPT over time [7-9, 16, 17].

b. Theory-translation: the clarification and formulation of core concepts by reference groups during methodological research leading to the development of toolkits $[18,19]$ and survey instruments [2022].

c. Theory-elaboration: development of new concepts during empirical studies and systematic reviews, that characterize and explain additional aspects of implementation processes [23-25].

Building a theory-informed framework for coding qualitative data involves a series of tasks that are, in themselves, a form of qualitative analysis. As we noted earlier, qualitative research focuses on the identification, characterization and interpretation of empirical regularities or deviant cases in natural language data. The process described here developed organically and opportunistically over a period of 18 months. The work of defining key concepts of the theory, assembling these into a framework, and then transforming them into a manual that can be used in practice, resulted from practically working on the concepts described below and making them fully usable. To do this, we drew on procedures suggested by Schreier [2] in her account of QCA. The general structure of coding manual development is laid out in Figure 1. 


\section{First pass selection and structuring.}

The result of the iterative development of NPY-like any theory-is a body of concepts representing the mechanisms that motivate and shape implementation processes, and also other features of interventions and the contexts in which their users make them workable and integrate them into practice. These core concepts of NPT were distributed over several papers that developed the theory, and in others that developed the means and methods of its application. In June 2020, these concepts were identified and assembled in a taxonomy of statements $(n=149)$. They took five main forms and are described in Table 1. This taxonomy consisted of concepts that defined, identified and characterized observable features of the collective action and collaborative work of implementation. Once prepared, it could be translated into a coding manual.

\section{Piloting the manual: first pass}

The taxonomy of 149 statements assembled in selection and structuring work included multiple duplicates, along ambiguous and overlapping descriptions of concepts. (These are described in the Online Supplementary Materials.) Duplicate and overlapping concepts were amalgamated, ambiguous concepts were either disambiguated or eliminated. After this work was competed, 38 discrete concepts were retained to make up a 'first pass' coding manual. (These are also described in the Online Supplementary Materials.) Earlier work to develop NPT, associated toolkits, and survey instruments had employed piloting of concepts and their definitions [17, 21,22, 26], to make sure that they could be used by researchers, practitioners, and policy makers. This manual was then applied to coding two papers selected from an earlier NPT systematic review. These were comprehensively coded and checked amongst the authors of this paper, who critically commented on coding decisions. The same coding manual was then applied to two bodies of interview data collected in other studies that were organized around NPT.

- A Randomized Controlled Trial. Transcripts of interviews ( $n=22$, with community mental health professionals) conducted for the process evaluation of the EYE-2 Trial (an engagement intervention for first episodes of psychosis employed in early intervention in the community [27],

- A 'real world' implementation program. Transcripts of interviews ( $n=55$ with managers, practitioners and patients) conducted for an evaluation of the accelerated implementation of remote clinicianpatient interaction in a tertiary orthopedic center during the Covid-19 pandemic.

This pilot work demonstrated that the main elements of the coding manual were workable in practice. However, it also suggested that some concepts still overlapped or were redundant. For example, a code that sought to outline 'events in processes' [28] that confounded implementation work, thus:

Intervention success: experienced workability and integration in contexts [29] 
In practice, this overlapped with

Internal (endogenous) processes and events that shape the capacity of participants to implement and operationalize interventions and their components (e.g. staff shortages, strategic initiatives) [9]

Intervention success was eliminated from the main body of the coding manual and moved to a 'holding' group of concepts, while Internal Processes was retained. Additionally, some concepts were insufficiently focused, and so were difficult to practically use. For example:

Coupling: Relations of interdependence between people, interventions and their components, and the contexts in which they are working. [10,30]

Less surprisingly, use of the coding frame suggested ways in which concepts were subject to different interpretations. Some of these were resolved by adjusting the wording in their definitions, but it was also clear that some concepts could be eliminated without affecting the integrity of the whole.

\section{Second pass selection and structuring}

The coding manual did characterize the core mechanisms that motivate and shape the work of implementation, along with concepts that represented the effects of this work. The piloting exercise revealed that the first pass coding manual was hard to use because it was over-complex and because it micro-managed the process of interpretation. This defeated attempts at nuanced interpretation. It was also clear that the shape of implementation processes themselves was much less visible in the coding manual. Here, the contexts or domains in which collective action and collaborative work, and their effects, are undertaken and experienced remained relatively invisible. This needed to be remedied.

In NPT, the contexts of implementation can be understood as both structures and processes. Following Hawe [28], we define contexts as events in systems that unfold over time within and between settings. To rectify the absence of a description of process domains, the taxonomy of NPT concepts was searched again for salient descriptors of context. Four main ways of framing implementation contexts were found:

i. Statements about implementation processes as contexts for the translation of strategic intentions into new or modified practices.

ii. Statements about implementation processes as contexts for making practices workable through adaptive execution.

iii. Statements about implementation processes as contexts for the integration of strategic intentions.

iv. Statements about implementation processes as organizational logics that are reframed as the collective action and collaborative work that characterizes implementation are accomplished.

As a result of second pass selection and structuring, concepts that represented contexts for action were added to the manual. These concepts are fully described in Table 2. 
- The four 'new' process concepts were then piloted 'in use' on a set of transcripts collected for an already completed study [31] of the design and implementation of treatment escalation plans to manage care at end of life in British hospitals.

It was found that they characterized process contexts effectively. These concepts were then presented and discussed in a series of international webinars in February-April 2021. Discussion with participants in those webinars assisted in finalizing the process model.

\section{Results: A Core Coding Manual For Normalisation Process Theory}

The final coding manual consisted of 12 primary NPT concepts, and 16 subsidiary concepts. Working through the procedures described above led to highly simplified form of NPT. Although it was not intended to do so, the final structure of the coding manual conforms to the Context-MechanismOutcomes configuration of realist evaluation studies [32]. It is organized thus:

- Contexts-events in systems unfolding over time within and between settingsin which implementation work is done. (Concepts are described in Table 2: strategic intentions; adaptive execution; negotiating capability; reframing organizational logic.)

- Mechanisms-made visible through collaborative work and collective action-that motivate and shapethe work that people do when they participate in implementation processes. (Concepts are described in Table 3: coherence-building; cognitive participation; collective action; reflexive monitoring.)

- Outcomes -the effects of implementation work in context-that make visible how things change as implementation processes proceed. (Concepts are fully described in Table 4: intervention performance; normative restructuring; relational restructuring; sustainment.)

Coding is a centrally important procedure in qualitative analysis, but it needs to be emphasized that it is only one part of a whole bundle of cognitive processes through which researchers make meanings in the data. For this reason, we recommend a layered approach to applying the coding manual. The coding manual itself is presented in Tables 2, 3, 4 and 5. It can be used in the following way.

\section{Familiarization and concept identification}

Familiarization. All qualitative analysis calls on researchers to become completely familiar with that part of the data-set that they wish to analyze. This may consist of any combination of original video or audiorecordings, fieldnotes, transcripts, social media posts, other documents, or already published texts.

Managing data. The organization of data analysis is of paramount importance. A set of agreed procedures needs to be developed for identifying, sharing and retrieving coded data. This may be done 
using software such as Atlas Ti, NVivo, or Dedoose); or by using a 'manual' method such as framework analysis on Microsoft Excel, or commentary using 'Track Changes' comments boxes in Microsoft Word. Both software and 'manual' methods can provide highly effective scaffolding for qualitative analysis.

Concept identification calls on the researcher to work through individual items in the data-set line by line and to link each item (which may be a phrase, sentence or paragraph in text, or an utterance or scene in untranscribed video or audio), either to one of the concepts included in the coding manual, or to a concept arising from some other source. In NPT studies these may be associated with constructs of the theory, and they may be related to the contexts, mechanisms, or outcomes of processes of adoption, implementation, and sustainment over time and between settings.

\section{Characterization and category-building}

Category-building calls on the researcher to use the definitions in the coding manual to identify the specific concepts or sub-concepts within each item in the data-set associated with Context, Mechanism, or Outcomes.

Characterization calls on the researcher to consider the relationship between, and significance of, concepts and sub-concepts. For example, are some constructs preconditions or barriers for others? Do others represent a temporal flow of events? Do the data suggest causal processes?

Identification of special cases. Within any qualitative data-set it is likely that there will be special cases. These may be particularly well-formed examples of phenomena that are typical of the data. Or, they may be what Glaser and Strauss call, 'deviant cases' [33], or what Tavory and Timmermans [34] call 'surprises', phenomena that are unexpected or very different within a data-set.

\section{Coding outside the manual.}

Familiarization and line by line analysis will inevitably reveal items in a data-set that cannot be easily categorized to the Context-Mechanism-Outcome model set out in the NPT Coding manual. Here, new categories can be also be created.

Associations between new and prior categories. Where new categories are associated with the categories already set out in the coding manual, it may be possible to treat them as sub-categories of these. For example, participants in implementation studies often talk about time as a personal or corporate resource, but these accounts are often

closely associated to material already coded under constructs such as Cognitive Participation or Relational Restructuring.

Differences between topics. Where new categories relate to a different topic, an extended coding manual that includes new categories can be created. These may be driven by engagement with data (e.g. an implementation study shows how the implementation of an intervention leads to experiences of gender- 
inequity and this is made into a parallel analytic track); or they may be driven by theory, (e.g. constructs from other theories can be used to identify and explore the whole range of inequities that can be created through an implementation process).

\section{Interpretation and explanation.}

Four NPT concepts related to mechanisms (Coherence-building, Cognitive Participation, Collective Action, Reflexive Monitoring), each possess four associated sub-concepts (described in Table 5). Users of NPT have often tended to treat these as discretionary in their coding and analysis [12-14], and their use is not mandatory. They do, however, add a layer of nuance to analysis of implementation mechanisms, and they remain highly usable and workable where the data support interpretation at that level of detail.

The act of coding is descriptive work that is a foundation for the interpretation of data, and is not a proxy for it. In each stage of coding, theoretical interpretations of the data can be recorded in what Glaser and Strauss [33] called Memos. These are commentaries on interpretations of the data and its contexts that the researcher makes as they perform coding.

It is not the purpose of a coding framework to verify the underpinning theory. The whole purpose of coding, and working abductively within theory, is to build and inform interpretation and understanding. This is not a discrete stage in data analysis but is continuous throughout. Researchers can hold joint 'data clinics' to surface these interpretive understandings, make necessary adjustments to the coding rules to capture the specificities of their data, work through their implications, and develop analytic propositions that add to, or extend, theory in relation to the specific phenomena of interest that their studies focus upon.

\section{Discussion}

Faced with a problem, people tend to add rather than subtract elements to produce a solution [6]. The purpose of developing this coding manual was to clarify and simplify NPT in use: to make it more workable, and to make it more easily integrated into research on the adoption, implementation, and use of sociotechnical and organizational innovations. In QCA-as in other forms of qualitative analysisproliferating constructs can easily make the business of coding ever more microscopic and less analytically rewarding. Indeed, the more parsimonious a prescheduled theoretical structure is, the more space it provides for nuanced interpretation and the development of novel categories of data and the analytic concepts that can be derived from them. In the development of an NPT coding manual described here, first pass selection and structuring sought to eliminate ambiguity and add workability at the outset, while second pass selection and structuring sought to eliminate overlapping or ambiguous constructs that were revealed in the analysis of qualitative data or in the coding of published articles. Taken together, these efforts yielded a set of 12 primary NPT concepts, and 16 sub-concepts. These concepts identify, characterize and explain the course of implementation processes through which strategic intentions are translated into practices, and enable understanding of how those practices can lead to different outcomes, and to varying degrees of sustainment. 


\section{Strengths and limitations}

We describe a set of methods likely to be useful be useful to qualitative researchers in other areas of research who wish to consider developing such manuals for other theories (for example, Relational Inequalities Theory [35], or Event System Theory [36]). A strength of the work was that developing the coding manual was undertaken by an international multidisciplinary team working with three recent empirical studies and a qualitative evidence synthesis. Regrettably, because this work was undertaken during the Covid-19 pandemic, we could not meet in person, and were forced to use zoom conference calls and email instead.

This ensured that the analytic process was closely embedded to the actual use of NPT. An important limitation of this work, though, is that it was undertaken opportunistically and grew organically. Development of a coding manual thus developed cumulatively, and authors joined the project, in an ad hoc way rather than being designed to do so from the outset. There might have been better ways to do this work, but it turned out to be a strength because it involved a continuous process of problem identification and solution.

\section{Conclusion}

Coding manuals are useful tools to support analytic work in qualitative research. They reduce cognitive load and at the same time render the assumptions underpinning qualitative analysis transparent and easily shared amongst teams of researchers. In this paper we have done three things. We have shown how a coding manual for the application of concepts proposed by Normalization Process Theory was developed, piloted, and made into a usable product. The coding manual performs two important tasks. It makes the application of NPT simpler for the user, and it links important NPT concepts to the practice of Realist Evaluation methods.

To our knowledge, this is the first qualitative coding manual for an implementation theory. It has been developed and used over a period of 18 months, and is already being used to inform practical implementation research in the field. It adds value to qualitative research on the adoption, implementation and sustainment of innovations by providing a stable, workable, set of concepts located in a well-established model of realist evaluation. It thus complements other resources for NPT researchers such as the NPT Toolkit and the NOMAD survey instrument [17, 21, 22, 26], and we have described the procedures by which researchers can make use of the coding manual. Finally, we have presented the manual in ready to use form.

\section{Abbreviations}

AKI Acute Kidney Injury

CDSS Computer Decision Support System 
CM Care Manager

CST Cognitive Stimulation Therapy

ERAS Enhanced Recovery After Surgery

FV Family Violence

GP General Practice

IPC Infection Prevention and Control

LGBT Lesbian, Gay, Bisexual and Transexual

$\mathrm{MCH}$ Mother and Child Health Nurse

MOVE Improving Maternal and Child Health Care for ㅁulnerable Mothers (Trial Acronym)

NPT Normalization Process Theory

OA Osteo-Arthritis

PO Physician Organizations

SDM Shared Decision-Making

\section{Declarations}

Ethics approval and consent to participate: No human subjects, animals or tissue were used in this research and no Ethical Approval was necessary to undertake it.

Consent for publication: Not applicable.

Availability of data and materials. All materials used in this research are included in this paper as tables, or are appended as online supplementary materials.

Competing interests: all authors have been involved in the development or use of Normalization Process Theory.

Funding: Contributions of CRM and EM were supported by NIHR North Thames Applied Research Collaborative. Contributions of TLF, TR, and SP were supported by NIHR North East and North Cumbria Applied Research Collaborative. The views expressed are those of the authors and not necessarily those of the NIHR or the Department of Health and Social Care.

Authors' contributions: Conception: CRM, TR. Design: CRM, AB, MB, TR, EM. Interpretation of data CRM, $B A, M B, T L F, A G, M G, K G, A M a c F, F S M, C M M, E M, S P$, TR; revision CRM, BA, MB, TLF, AG, MG, KG, AMacF, 
FSM, CMM, EM, SP, TR; Approved the submitted version of this paper CRM, BA, MB, TLF, AG, MG, KG, AMacF, FSM, CMM, EM, SP, TR

\section{References}

1. Strauss A: Qualitative analysis for social scientists. Cambridge: Cambridge University Press; 1987.

2. Schreier M: Qualitative Content Analysis in Practice. London: SAGE; 2012.

3. Hsieh H-F, Shannon SE: Three approaches to qualitative content analysis. Qual Health Res 2005, 15(9):1277-1288.

4. Patton MQ: Two decades of developments in qualitative inquiry: A personal, experiential perspective. Qual Social Work 2002, 1(3):261-283.

5. Mclellan-Lemal K, MacQueen E: Team-based codebook development: Structure, process, and agreement. In: Handbook for team-based qualitative research. Edited by Guest, G. Altamira, Lanham MD. 2008:119-136

6. Adams GS, Converse BA, Hales AH, Klotz LE: People systematically overlook subtractive changes. Nature 2021, 592(7853):258-261.

7. May C: A rational model for assessing and evaluating complex interventions in health care. $B M C$ Health Serv Res 2006, 6(86):1-11.

8. May C, Finch T: Implementing, embedding, and integrating practices: an outline of normalization process theory. Sociology 2009, 43(3):535-554.

9. May C: Towards a general theory of implementation. Implement Sci 2013, 8(1):18.

10. May CR, Johnson M, Finch T: Implementation, context and complexity. Implement Sci 2016, 11(1):141.

11. May C, Rapley T, Finch T: Normalization Process Theory. In: International Handbook of Implementation Science. Edited by Nilsen P, Birken S. London: Edward Elgar; 2020: 144-167.

12. McEvoy R, Ballini L, Maltoni S, O'Donnell CA, Mair FS, MacFarlane A: A qualitative systematic review of studies using the normalization process theory to research implementation processes. Implement Sci $2014,9$.

13. May CR, Cummings A, Girling M, Bracher M, Mair FS, May CM, Murray E, Myall M, Rapley T, Finch T: Using Normalization Process Theory in feasibility studies and process evaluations of complex healthcare interventions: a systematic review. Implement Sci 2018, 13(1):80.

14. Huddlestone L, Turner J, Eborall H, Hudson N, Davies M, Martin G: Application of normalisation process theory in understanding implementation processes in primary care settings in the UK: a systematic review. BMC Fam Pract 2020, 21(1):1-16.

15. Mohr JW, Wagner-Pacifici R, Breiger RL: Toward a computational hermeneutics. Big Data \& Society 2015, 2(2):2053951715613809.

16. May C, Finch T, Mair F, Ballini L, Dowrick C, Eccles M, Gask L, MacFarlane A, Murray E, Rapley T.: Understanding the implementation of complex interventions in health care: the normalization 
process model. BMC Health Serv Res 2007, 7(148).

17. May C, Mair FS, Finch T, MacFarlane A, Dowrick C, Treweek S, Rapley T, Ballini L, Ong BN, Rogers A et al: Development of a theory of implementation and integration: Normalization Process Theory. Implement Sci 2009, 4(29)

18. Murray E, May C, Mair F: Development and formative evaluation of the e-Health Implementation Toolkit (e-HIT). BMC Med Inform Decis 2010, 10(1):61.

19. May C, Finch T, Ballini L, MacFarlane A, Mair F, Murray E, Treweek S, Rapley T: Evaluating Complex Interventions and Health Technologies Using Normalization Process Theory: Development of a Simplified Approach and Web-Enabled Toolkit. BMC Health Serv Res 2011, 11(1):245.

20. Finch TL, Rapley T, Girling M, Mair FS, Murray E, Treweek S: Improving the normalization of complex interventions: measure development based on normalization process theory (NoMAD): study protocol. Implement Sci 2013, 8.

21. Rapley T, Girling M, Mair FS, Murray E, Treweek S, McColl E, Steen IN, May CR, Finch TL: Improving the normalization of complex interventions: part 1 - development of the NoMAD instrument for assessing implementation work based on normalization process theory (NPT). BMC Med Res Methodol 2018, 18(1):133.

22. Finch TL, Girling M, May CR, Mair FS, Murray E, Treweek S, McColl E, Steen IN, Cook C, Vernazza CR et al: Improving the normalization of complex interventions: part 2 - validation of the NoMAD instrument for assessing implementation work based on normalization process theory (NPT). BMC Med Res Methodol 2018, 18(1):135.

23. Mair FS, May C, O'Donnell C, Finch T, Sullivan F, Murray E: Factors that promote or inhibit the implementation of e-health systems: an explanatory systematic review. Bull World Health Organ 2012, 90(5):357-364.

24. May C, Sibley A, Hunt K: The nursing work of hospital-based clinical practice guideline implementation: An explanatory systematic review using Normalisation Process Theory. Int J Nurs Stud 2014, 51(2):289-299.

25. Johnson MJ, May CR: Promoting professional behaviour change in healthcare: what interventions work, and why? A theory-led overview of systematic reviews. BMJ open 2015, 5(9):e008592.

26. May CR, Finch T, Ballini L, MacFarlane A, Mair F, Murray E, Treweek S, Rapley T: Evaluating complex interventions and health technologies using normalization process theory: development of a simplified approach and web-enabled toolkit. BMC Health Serv Res 2011, 11.

27. Greenwood K, Webb R, Gu J, Fowler D, de Visser R, Bremner S, Abramowicz I, Perry N, Clark S, O’Donnell A et al: The Early Youth Engagement in first episode psychosis (EYE-2) study: pragmatic cluster randomised controlled trial of implementation, effectiveness and cost-effectiveness of a team-based motivational engagement intervention to improve engagement. Trials 2021, 22(1):272

28. Hawe P, Shiell A, Riley T: Theorising Interventions as Events in Systems. Am J Commun Psychol 2009, 43(3-4):267-276. 
29. May C: Agency and implementation: Understanding the embedding of healthcare innovations in practice. Soc Sci Med 2013, 78(0):26-33.

30. Perrow C: Normal accidents : living with high-risk technologies. New York: Basic Books; 1984.

31. May C, Myall M, Lund S, Campling N, Bogle S, Dace S, Richardson A: Managing patient preferences and clinical responses in acute pathophysiological deterioration: What do clinicians think treatment escalation plans do? Soc Sci Med 2020, 258:113143.

32. Pawson R, Tilley N: Realistic evaluation. London: Sage Publications; 1997.

33. Glaser BG, Strauss A: The discovery of grounded theory. Chicago: Aldine; 1967.

34. Tavory I, Timmermans S: Abductive Analysis: Theorizing Qualitative Research: University of Chicago Press; 2014.

35. Avent-Holt D, Tomaskovic-Devey D: Organizations as the building blocks of social inequalities. Sociol Compass 2019, 13(2).

36. Morgeson FP, Mitchell TR, Liu D: Event System Theory: An Event-Oriented Approach to the Organizational Sciences. Acad Manage Rev 2015, 40(4):515-537.

37. Ong BN, Morden A, Brooks L, Porcheret M, Edwards JJ, Sanders T, Jinks C, Dziedzic K: Changing policy and practice: making sense of national guidelines for osteoarthritis. Social Sci Med 2014, 106:101-109.

38. Pope C, Halford S, Turnbull J, Prichard J, Calestani M, May C: Using computer decision support systems in NHS emergency and urgent care: ethnographic study using normalisation process theory. BMC Health Serv Res 2013, 13(1):111.

39. Agreli H, Barry F, Burton A, Creedon S, Drennan J, Gould D, May CR, Smiddy MP, Murphy M, Murphy $S$ et al: Ethnographic study using Normalization Process Theory to understand the implementation process of infection prevention and control guidelines in Ireland. BMJ Open 2019, 9(8):e029514.

40. Sutton E, Herbert G, Burden S, Lewis S, Thomas S, Ness A, Atkinson C: Using the normalization process theory to qualitatively explore sense-making in implementation of the enhanced recovery after surgery programme: "it's not rocket science". PLOS ONE 2018, 13(4).

41. Dickinson C, Gibson G, Gotts Z, Stobbart L, Robinson L: Cognitive stimulation therapy in dementia care: exploring the views and experiences of service providers on the barriers and facilitators to implementation in practice using Normalization Process Theory. International Psychogeriatrics 2017, 29(11):1869-1878.

42. Jones $\mathrm{CH}$, Glogowska M, Locock L, Lasserson DS: Embedding new technologies in practice-a normalization process theory study of point of care testing. BMC Health Serv Res 2016, 16(1):591.

43. Trietsch J, van Steenkiste B, Hobma S, Frericks A, Grol R, Metsemakers J, van der Weijden T: The challenge of transferring an implementation strategy from academia to the field: a process evaluation of local quality improvement collaboratives in Dutch primary care using the normalization process theory. J Eval Clin Pract 2014, 20(6):1162-1171. 
44. Røsstad T, Garåsen H, Steinsbekk A, Håland E, Kristoffersen L, Grimsmo A: Implementing a care pathway for elderly patients, a comparative qualitative process evaluation in primary care. $B M C$ Health Serv Res 2015, 15(1).

45. Foster M, Burridge L, Donald M, Zhang J, Jackson C: The work of local healthcare innovation: A qualitative study of GP-led integrated diabetes care in primary health care Organization, structure and delivery of healthcare. BMC Health Serv Res 2016, 16(1).

46. Overbeck G, Davidsen AS, Kousgaard MB: Enablers and barriers to implementing collaborative care for anxiety and depression: A systematic qualitative review. Implement Sci 2016, 11(1).

47. Hall A, Wilson CB, Stanmore E, Todd C: Implementing monitoring technologies in care homes for people with dementia: A qualitative exploration using Normalization Process Theory. Int $J$ Nurs Stud 2017, 72:60-70.

48. May C, Finch T: Implementation, embedding, and integration: an outline of Normalization Process Theory. Sociology 2009, 43(3):535-554.

49. Keenan J, Poland F, Manthorpe J, Hart C, Moniz-Cook E: Implementing e-learning and e-tools for care home staff supporting residents with dementia and challenging behaviour: A process evaluation of the ResCare study using normalisation process theory. Dementia 2018.

50. Alharbi TS, Carlström E, Ekman I, Olsson L-E: Implementation of person-centred care: management perspective. J Hosp Admin 2014, 3(3):p107.

51. Morden A, Brooks L, Jinks C, Porcheret M, Ong BN, Dziedzic K: Research "push", long term-change, and general practice. J Health Organ Management 2015, 29(7):798-821.

52. Lloyd A, Joseph-Williams N, Edwards A, Rix A, Elwyn G: Patchy 'coherence': using normalization process theory to evaluate a multi-faceted shared decision making implementation program (MAGIC). Implement Sci 2013, 8:102.

53. Valaitis R, Cleghorn L, Dolovich L, Agarwal G, Gaber J, Mangin D, Oliver D, Parascandalo F, Ploeg J, Risdon C: Examining Interprofessional team structures and processes in the implementation of a primary care intervention (Health TAPESTRY) for older adults using normalization process theory. BMC Fam Pract 2020, 21.

54. Burau V, Carstensen $K$, Fredens $M$, Kousgaard MB: Exploring drivers and challenges in implementation of health promotion in community mental health services: A qualitative multi-site case study using Normalization Process Theory. BMC Health Serv Res 2018, 18(1).

55. Asiedu GB, Fang JL, Harris AM, Colby CE, Carroll K: Health Care Professionals' Perspectives on Teleneonatology Through the Lens of Normalization Process Theory. Health Sci Rep 2019, 2(2):e111.

56. Shulver W, Killington M, Crotty M: 'Massive potential'or 'safety risk'? Health worker views on telehealth in the care of older people and implications for successful normalization. BMC Med Inform Dec Mak 2016, 16(1):131.

57. Hooker L, Small R, Humphreys C, Hegarty K, Taft A: Applying normalization process theory to understand implementation of a family violence screening and care model in maternal and child 
health nursing practice: a mixed method process evaluation of a randomised controlled trial. Implement Sci 2015, 10(1):39.

58. Ziegler E, Valaitis R, Yost J, Carter N, Risdon C: "Primary care is primary care": Use of Normalization Process Theory to explore the implementation of primary care services for transgender individuals in Ontario.PLOS ONE 2019, 14(4):e0215873.

59. Holtrop JS, Potworowski G, Fitzpatrick L, Kowalk A, Green LA: Effect of care management program structure on implementation: a normalization process theory analysis. BMC Health Serv Res 2016, 16(1):386.

60. Scott J, Finch T, Bevan M, Maniatopoulos G, Gibbins C, Yates B, Kilimangalam N, Sheerin N, Kanagasundaram NS: Acute kidney injury electronic alerts: mixed methods Normalisation Process Theory evaluation of their implementation into secondary care in England. BMJ Open 2019, 9(12):e032925.

61. Bamford C, Poole M, Brittain K, Chew-Graham C, Fox C, lliffe S, Manthorpe J, Robinson L, team C: Understanding the challenges to implementing case management for people with dementia in primary care in England: a qualitative study using Normalization Process Theory. BMC Health Serv Res 2014, 14(1):549.

\section{Tables}

Table 1 Typology of NPT Statements 


\section{Statement Example \\ typology}

Statements derived from other theories that had been 'federated' with NPT.
'Individual intentions: agents' readiness to translate individual beliefs and attitudes into behaviors that are congruent, or not congruent, with system norms and roles. They frame individual motivation to participate in a complex intervention' [9].
Statements defining the work of implementation.
'By normalization, we mean the work that actors do as they engage with some ensemble of activities (that may include new or changed ways of thinking, acting, and organizing) and by which means it becomes routinely embedded in the matrices of already existing, socially patterned, knowledge and practices '[8].
Statements about relationships between actors, interventions, and contexts.
'The more tightly coupled intervention components are, the less discretion participants have in resource mobilisation and collective action, and the more they must do adaptive work to ensure intervention integration' [10].
NPT

Propositions.
'A complex intervention is disposed to normalization if it equals or improves accountability and confidence within networks' [7].
Statements about mechanisms of action.
'Collective action through which effort is invested in an intervention and its components: participants mobilize skills and resources and make a complex intervention workable. This work frames how participants realize and perform intervention components in practice' [11].

\section{Table 2: Contexts: domains in which implementation work is done}




\section{Construct Source reference, and example text}

Strategic

intentions
Contexts (patterns of social relations, environments, and events unfolding over time and across settings) in which an intervention can be formulated and plans for enacting it can be put in train [11].

Example: 'The analysis centres on English primary care and in particular on the issue of how healthcare professions are affected by, and in turn affect, the interpretation and adoption of new services. We use the case of the implementation of evidence-based approaches for managing patients with osteoarthritis. This musculoskeletal problem occurs in a high proportion of GP consultations, and is projected to increase due to a rapidly ageing population in the western world' [37].
Adaptive execution

Contexts (patterns of social relations, environments, and events unfolding over time and across settings) in which an intervention can be made workable [11].

Example: 'Huge effort was expended and continues to be required to implement and keep this technology in use. This innovation must be understood both as a computer technology and as a set of practices related to that technology, kept in place by a network of actors in particular contexts. While technologies can be 'made to work' in different settings, successful implementation has been achieved, and will only be maintained, through the efforts of those involved in the specific settings and if the wider context continues to support the coherence, cognitive participation, and reflective monitoring processes that surround this collective action. Implementation is more than simply putting technologies in place - it requires new resources and considerable effort, perhaps on an on-going basis' [38].

Negotiating capacity
Contexts (patterns of social relations, environments, and events unfolding over time and across settings) in which an intervention can be integrated [11].

Example: 'Aligning IPC guidelines with local clinical context is an essential means to reduce the sense of dissonance and represents a critical step forward towards successful implementation. Some strategies described in the literature to promote alignment include: integration of IPC recommendations within other established programmes; and education and audit interventions acknowledging the positive and negative beliefs of staff on IPC practices [39].
Reframing organizational logics
Contexts (patterns of social relations, environments and events unfolding over time and across settings) that are expected to be changed by an intervention [11].

Example: 'The external and internal partnership building were key and also strategic, so as not to impose ERAS but to co-create it from the ground up. This relational work, as framed in the NPT, is deceptively complex as it involves convincing others that this is a legitimate improvement programme worth participating in without devaluing their current practice and beliefs. The interprofessional and 
interdepartmental relationships the champion teams established appeared to lay an important foundation for accepting changes and the data reports as meaningful and embedding ERAS into everyday practice' [40].

Table 3: Mechanisms: the work that people do to make implementation happen. 


\section{Construct Source reference, and example text}

Coherence Coherence-building is collaborative work that makes interventions and their

building components meaningful. Participants work to understand how intervention components are different from other practices, and they work to make them a coherent proposition for action [11].

Example: 'Coherence was achieved around the CDSS despite local context variation. Across all three sites there was agreement that the CDSS was suitable for the (varied) tasks and that appropriate resources were in place to enable effective implementation, although these varied between settings. There were differences between settings where the CDSS replaced an established system with existing staff and where the service and/or the staff were new and the work of establishing coherence had to be altered to reflect this. It was clear that knowledge, experience and work identities built through doing call-handling work influenced the coherence of the CDSS for staff in the different settings. What is especially interesting in the wider policy context - where this same CDSS is now being used to support a national ' 111 ' urgent care service (...) is that coherence was not just a local 'problem', it was necessarily underpinned by wider understandings and discourses for example about the necessity of rationing and the need to modify caller/patient behaviour and beyond that the very legitimacy of evidence based medicine and the kinds of expert knowledge which underpinned the CDSS' [38]

Cognitive participation
Cognitive participation forms commitment around an intervention and its components. This establishes its legitimacy and enrolls themselves and others into an implementation process. This work frames how participants become members of a specific community of practice [11]

Example: 'Cognitive participation relates to the work that participants undertake to build up and sustain a community of practice around an intervention. In terms of CST, participants identified training as an important factor in generating their own and their colleagues' interest in CST and thus ensuring all stakeholders were involved. Staff were further motivated to continue running the groups within their service through observing the direct beneficial effects of CST on clients' [41].
Collective Action
People work collaboratively to realize, perform, and operationalize and intervention and its components [11].

Example: 'The daily tasks involved in carrying out Point of Care $(\mathrm{POC})$ testing were deciding which tests (if any) to take for each patient when they arrived; communicating this to others; taking the blood; running the tests; examining the results; communicating the results to others; and deciding what action to take accordingly. This work was allocated to different staff according to their skills and availability. Close teamwork appeared key to ensuring that each task was performed by an appropriate person at the necessary time' [42]. 
monitoring components, and utilize that knowledge to reconfigure social relations and action [34].

Example: 'Data provision by the laboratories proved to be difficult despite the standardized format. The database manager at the central level reported he had to put much effort in getting the data from the system administrator from the laboratories because they did not prioritize data delivery. It was reported by them that saving the data extraction queries, as the research group suggested, for use in the next time period was increasingly helpful in the course of the implementation period. By fine-tuning these queries after each extraction, the quality of the delivered data improved' [43].

Table 4: Outcomes: what are the results of implementation work? 


\section{Construct Source reference, and example text}

Sustainment Sustainment activities through which the normalization of an intervention and its embedding in everyday practice are carried forward as continuous translational action [11].

Example: 'At the end of the project period, the pathway was integrated in daily practice in two of the six municipalities. In these municipalities the care pathway was found to have the potential of structuring the provision of home care services and collaboration with the GPs, and serving as a management tool to effect change and improve knowledge and skills. (...) The generic care pathway for elderly patients has a potential of improving follow-up in primary care by meeting professional and managerial needs for improved quality of care, as well as more efficient organisation of home care services. However, implementation of this complex intervention in fulltime running organisations was demanding and required’ [44].

Normative restructuring
Changes to norms, rules and resources that happen as a result of working with interventions and their components [10].

Example: 'The first theme, trusting and embedding new relationships, is a reminder that while locally-led innovation is designed to address local problems, convincing others of its value is core work. This is particularly so when the innovation challenges professional norms and involves changes to traditional delivery models and renegotiation of professional roles (...). In this case, the findings are consistent with previous research which has indicated that the success of such innovations is dependent on the trust of all involved and the credibility of

clinicians (...)' [45].

Relational restructuring

Changes to the ways that people are organized and relate to each other that happen as a result of working with interventions and their components [44].

Example: 'The CMs became "everyday representatives" for the secondary sector and were responsible for acting as bridge-builders between hospital psychiatry and general practice. Previous research on Nurse Practitioners/ Advanced Nurse Practitioners in general practice (...) has shown that if the clinics are not involved at an early stage and prepared thoroughly for the Nurse Practitioner's arrival, their integration in general practice is hampered. Preparation involves practical issues, a clearly defined role for the nurse practitioner, and organizational leadership, meaning that the managers of the responsible organization must be involved in the process of defining and supporting the role (...) The challenges also pointed towards a lack of managerial co- ordination of, and responsibility for, the practical issues associated with the CM's role in general practice. (...) This meant that on many occasions, the $\mathrm{CMs}$ had to take on the role of implementation ambassadors assuming responsibility for maintenance of the collaborative care model' [46]. 
over time and across settings. The mobilization of these ensembles may be highly variable [6]

Example: 'The bed-monitoring technologies were felt to be useful in helping staff identify patterns in resident behaviour and explore reasons for these behaviours. The bed sensors at Sycamore Lane were capable of recording clinical data such as heart rate, but the manager reported that "it's not something that we use readily", and this functionality was never observed in use during the present study. The location-based system at Conifer Gardens was similarly able to record data, including information about resident mobility activity. This functionality had initially been anticipated as potentially useful for enhancing clinical understanding, however, the Occupational Therapist reflected that the time needed to analyse and interpret these data had been "a job in itself" and thus has been difficult to integrate into daily practice. There were questions about the clinical utility of some of the data, which appeared to become more pronounced when considering the financial expense of the technology' [47].

Table 5: Mechanisms of action: NPT Sub-concepts 


\begin{tabular}{|lll}
$\begin{array}{l}\text { NPT } \\
\text { construct }\end{array}$ & $\begin{array}{l}\text { Sub-construct } \\
\text { and source } \\
\text { references }\end{array}$ & Verbatim extracts from example texts \\
\hline
\end{tabular}

Coherence

\section{Differentiation: \\ An important \\ element of \\ sense-making \\ work is to \\ understand how \\ interventions \\ and their \\ components, \\ and prior \\ practice are \\ different from \\ each other [48].}

'In order to invest in ERAS individuals needed to be able to differentiate its practices favourably with those enacted preimplementation. This required coherence work in understanding the potential patient benefits allied to its introduction. Participants provided divergent accounts when they compared ERAS to previous practice. A number of participants asserted that the introduction of ERAS had brought about considerable changes to their day-to-day practice. These changes included positive adjustments in the management of patients and required patients to play a more active role in their own recovery' [40].

\section{Communal specification: Sense-making relies on people working together to build a shared understanding of the aims, objectives, and expected benefits of interventions and their components [48].}

'Another barrier to coherence was lack of communal specification, since not everyone considered they had been informed about the study or understood its aims and processes. This caused implementation problems for the homes and the research team. For the homes, the researchers' reasons for examining potential benefits from the intervention to have a positive impact on the culture of care had not been strongly reflected' [49].

\section{Individual specification: Sense-making has an individual component, too. Here participants in coherence work need to do things that will help them understand their specific tasks and responsibilities around interventions and their components [48].}

Internalization: Sense-making involves people
'One respondent felt discussing the new way to view the patients with the staff was a delicate issue. In the old care model, patients were usually only informed about the treatment whilst now, in the care model, patients were to be seen as partners. This was regarded as a shift in power and, at least for some physicians, it would be difficult to get used to' [50]. 
in work that is

about

understanding

the value,

benefits and

importance of

interventions

and their

components

[48].
Initiation: A core

problem is

whether or not

key participants

are working to

drive

interventions

and their

components.

forward. [48]

\section{Enrolment:}

Participants

may need to

organize or

reorganize

themselves and

others in order to

collectively

contribute to the

work involved in

interventions

and their

components

[48].

Legitimation: An

important

component of

relational work

around

interventions

and their

components is

the work of

ensuring that

other

participants

believe it is right

for them to be

involved, and

that they can

make a valid

contribution to it

[48]. their own interests. The GPs in General Practice 8 provided their views at the end of the introductory meeting, saying that they liked the structure and more systematic approach to caring for people with OA and concluded that "it is nice to be able to try something that may make a difference"' [51].
'Participants described the new SDM work as requiring leaders to define the work, and then enrolling others to contribute collectively to the process. Identifying leadership support for SDM was challenging: clinical teams are not simple hierarchical units, and substantial autonomy exists, especially for experienced clinicians' [52].
'Clinic participants also re- ported that the intervention provided a model for improved interprofessional team collaboration, resulting in a greater understanding of clinicians' roles and skill sets. Huddles were viewed as worth creating and maintaining, both for interprofessional team and patient benefits.

Participants identified that the majority of patients were satisfied with the interprofessional approach to primary care' [53].
Activation: Once it is underway, participants
'The respondents offered several explanations for resistance or lack of engagement: some staff felt that health promotion activities overstretched users' resources and thus had a negative impact on their quality of life; others argued that health promotion activities did not respect personal preferences of users and staff (...) One of the important implementation ideas (...) was the concept of staff being role models for health promotion. As role models staff were[ expected to participate in different health promotion activities (like joining users for walks and meals) and to display a healthy lifestyle at work. In the four providers, such expectations were formulated and formalised by management or by key implementation staff to different extents. However, in all cases some staff did not buy into this idea; they felt that the elements of smoking cessation and healthier meals interfered with their usual lifestyle and personal preferences' [54].
'While, overall, this system has worked well, many participants referenced instances of long wait times and rerouting of calls to reach the neonatologist. Based on the care teams' appraisal and 
need to

collectively

define and enact

the actions and

procedures

needed to

sustain

interventions

and their

components and

to stay involved

[48].

\section{Collective Action}

experience with this process, they suggested modeling the teleneonatology service activation after the emergency department's response system, for immediate and direct connection. Other suggestions include making the technology simple enough for ease of use, and to mount a camera (which can be controlled by the remote neonatologist) to the baby warmer' [55].

\section{Interactional Workability: This} refers to the interactional work that people do with each other, and with the components of interventions and their components when they seek to operationalize them in everyday settings [7].
'The rural allied health team indicated that telehealth technology provided 'a whole range of other capabilities', and considered it 'safe and it's appropriate and it's an equivalent, if not better, sort of service that you can provide'. They were committed to the notion that telehealth could balance the unequal access to services across geographical locations, and were keen to pursue innovative ways of using telehealth technologies to allow them to provide complex distant therapy. In contrast to rural and experienced telehealth clinicians who were keen to utilise technology as part of their role and to deal with distance and isolation, urban clinicians with no exposure to telehealth reported more reservations about the safety and suitability of providing rehabilitation through telehealth. They generally felt that telehealth should be reserved for 'people who are more autonomous and more capable and ... straightforward', rather than 'real' rehabilitation patients with complex issues. They felt that people who required rehabilitation often require a 'hands on' approach' [56].

\section{Relational Integration: This refers to the knowledge work that people do to build accountability and maintain confidence in interventions and their components and in each other as they use them [7].}

'Enhanced collegial discussion about FV and adherence to the safety measures, such as the home visiting policy and procedures introduced in (...) model, were important for nurses to feel safe and undertake the FV work. As implementation progressed, intervention nurses felt safer than comparison nurses when attending home visits (...). Relationships within teams and with FV services varied across the $\mathrm{MCH}$ intervention teams. High workloads, time constraints and a lack of nursing staff or relievers in some centres impacted on the organisation of the FV work at times. The nurse mentor role to provide secondary consultation, linkage to FV services and support for other $\mathrm{MCH}$ nurses had varied success. Due to time constraints and the often solo nature of $\mathrm{MCH}$ practice, most nurses preferred to discuss clinical issues with a nurse friend or coworker at the time rather than try to contact the designated MOVE nurse mentor, with only $38 \%$ of nurses using the nurse mentor role early in the trial. This increased to $52 \%$ as time went on. If the nurse was not comfortable speaking and had insufficient time or access to the nurse mentor, then this aspect of the model was lost' [57].

\section{Skill set Workability: This refers to the}

'A key theme identified in the literature and through this study is the need for more training for practitioners. This includes training both in professional education and continuing 
allocation and training work that underpins that is built up around interventions and their components as it is operationalised in the real world [7]. educational opportunities for all practitioners. Medical, nursing and allied health education programs need to improve LGBT curriculum content $[2,64-66]$. Providing education on general terminology, healthcare needs specific to the transgender population, and practitioners' role in providing healthcare for this population will better prepare new practitioners for serving this community. Increased access to continuing education with LGBT content will help to increase the knowledge and skill of current practitioners. Embedding LGBT content within current programs of continuing education may increase awareness more than having specific LGBT courses (...). Embedding it in current programs may bring awareness to the concepts and highlight the need for practitioners to seek out more specific training to address their learning gaps' [58].

\section{Contextual Integration: This refers to the resource work - supporting interventions and their components through the allocation of different kinds of resources and the execution of protocols, policies and procedures [7].}

'Since POs were able to self-select into the pilot, the alignment of $\mathrm{PO}$ priorities with participation in a pilot on care management was a good fit. The leadership in all POs voiced interest in providing care management to patients within their $\mathrm{PO}$ as a means of improving patient outcomes, easing burden on providers of handling complex patients, and to meet health care standards and reimbursement policies such as patient-centered medical home recognition, accountable care, and meaningful use. Therefore, in this study overall organizational support was not found to be variant. Where organizational support emerged as an issue related more to resources and support for the care management program relative to the needs and goals of the program. The most common issue here was not having either enough care managers or enough care manager protected time to do care management for the number of patients needing it. So in well-normalized programs, there was a sense of "rationing" of the care manager. Because the program was being used so much more and there was a capacity constraint at the practice level with the practice-based care manager structure, the practices in these POs voiced more concern about lack of care manager capacity. Lack of resources was evident in other ways such as lack of space for patient visits or access to phone lines to make longer calls' [59].

\section{Reflexive Monitoring}

\author{
Systematization: \\ participants in \\ interventions \\ and their \\ components \\ may seek to \\ determine how \\ effective and \\ useful it is for \\ them and for \\ others, and this \\ involves the \\ work of \\ collecting \\ structured \\ information in a
}

'Feedback was never provided to staff on the effect of the AKI ealert "I haven't had any feedback since the new version (of the AKI e-alert) went in actually(...) I don't know whether there is a formal mechanism for that getting to anyone"' [60]. 


\section{Communal appraisal:}

participants

work together -

sometimes in

formal

collaboratives,

sometimes in

informal groups

to evaluate the

worth of

interventions

and their

components

They may use

many different

means to do this

drawing on a

variety of

experiential and

systematized

information [48].

\section{Individual}

appraisal:

Participants in

interventions

and their

components

also work

experientially as

individuals to

appraise its

effects on them

and the contexts

in which they are

set. From this

work stem

actions through

which

individuals

express their

personal

relationships to

new

technologies or

complex

interventions

[48].

Reconfiguration: appraisal work by individuals or groups may lead to attempts to redefine procedures or
'The e-alert was rarely (if ever) discussed among clinicians, but participants often stated they felt that others would find it worthwhile. "The e-alert was rarely (if ever) discussed among clinicians, but participants often stated they felt that others would find it worthwhile. "Most people I'm sure would know it's a good idea having them. That's what l'd say to someone about these alerts"'[60].

'A key barrier which has not previously been identified concerned the ability of case managers to identify, and act on, emerging patient and carer needs; we identified examples of missed and unmet needs for all three case managers. One case manager explicitly attributed this to the timing of the intervention; a study of case management for people with early symptoms of dementia and their carers similarly found that case managers did not feel the intervention was needed at this point' [61].
'Aligning IPC guidelines with local clinical context is an essential means to reduce the sense of dissonance and represents a critical step forward towards successful implementation. Some strategies described in the literature to promote alignment include: integration of IPC recommendations within other established programmes; and education and audit interventions 
modify

interventions

and their

components

[48].

\section{Figures}

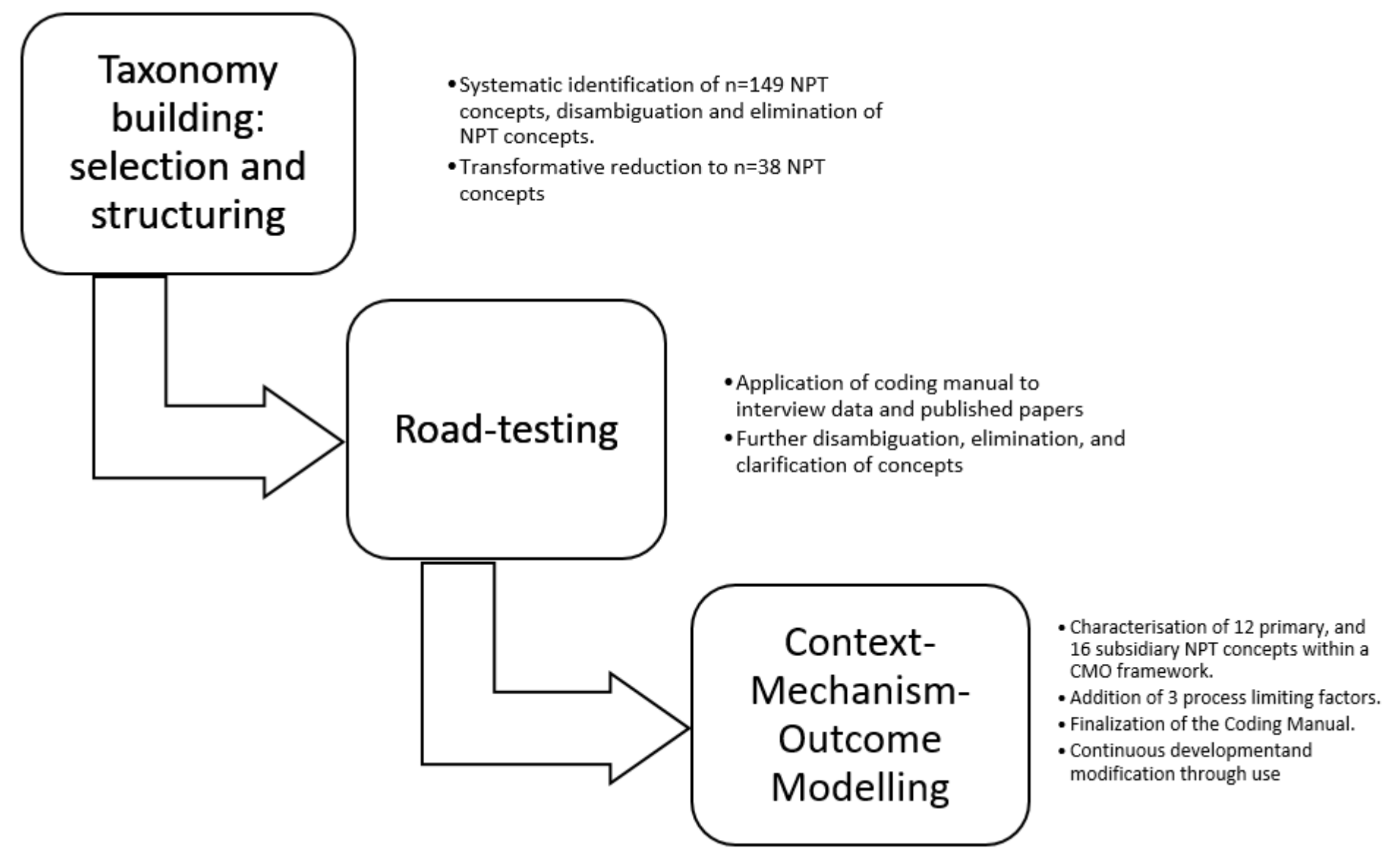

Figure 1

Simplified model of Coding Manual development procedures

\section{Supplementary Files}

This is a list of supplementary files associated with this preprint. Click to download.

- ONLINESUPPLEMENTARYMATERIAL.docx 